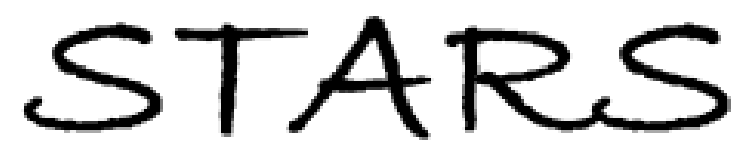

University of Central Florida

STARS

$1-1-2006$

\title{
Genetically engineered human mesenchymal stem cells produce met-enkephalin at augmented higher levels in vitro
}

Ikuko Sugaya

University of Central Florida

Tingyu Qu

Kiminobu Sugaya

University of Central Florida

George D. Pappas

Find similar works at: https://stars.library.ucf.edu/facultybib2000

University of Central Florida Libraries http://library.ucf.edu

This Article is brought to you for free and open access by the Faculty Bibliography at STARS. It has been accepted for inclusion in Faculty Bibliography 2000 s by an authorized administrator of STARS. For more information, please contactSTARS@ucf.edu.

\section{Recommended Citation}

Sugaya, Ikuko; Qu, Tingyu; Sugaya, Kiminobu; and Pappas, George D., "Genetically engineered human mesenchymal stem cells produce met-enkephalin at augmented higher levels in vitro" (2006). Faculty Bibliography 2000s. 6621.

https://stars.library.ucf.edu/facultybib2000/6621

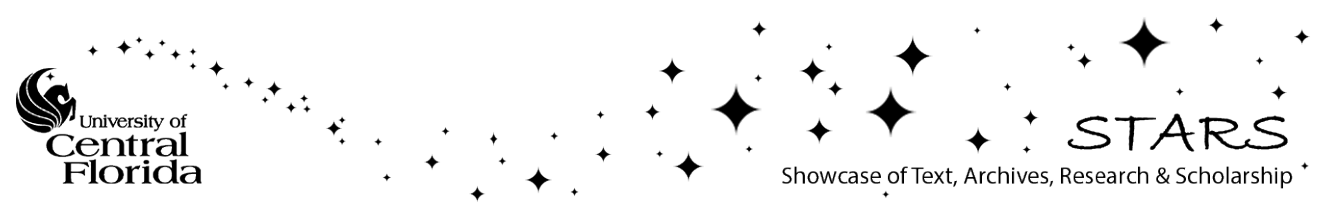




\title{
Genetically Engineered Human Mesenchymal Stem Cells Produce Met-Enkephalin at Augmented Higher Levels In Vitro
}

\author{
Ikuko Sugaya,* Tingyu Qu, $\dagger$ Kiminobu Sugaya,* and George D. Pappas $\dagger$ \\ *Biomolecular Science Center, Burnett College of Biomedical Sciences, \\ University of Central Florida, Orlando, FL 32816-2364, USA \\ $\dagger$ The Psychiatric Institute, Department of Psychiatry, and Department of Anatomy and Cell Biology, \\ University of Illinois at Chicago, Chicago, IL 60612, USA
}

\begin{abstract}
We have reported that transplantation of adrenal medullary chromaffin cells that release endogenous opioid peptides into pain modulatory regions in the CNS produce significant antinociceptive effects in patients with terminal cancer pain. However, the usefulness of this procedure is minimal because the availability of human adrenal tissue is very limited. Alternative xenogeneic materials, such as porcine and bovine adrenal chromaffin cells present problems of immune rejection and possible pathogenic contamination. In an attempt to develop opioid peptide-producing cells of autologous origin, we have transfected human mesenchymal stem cells (hMeSCs) with a mammalian expression vector containing a fusion gene of green fluorescent protein (GFP) and human preproenkephalin (hPPE), a precursor protein for enkephalin opioid peptides. Enkephalins are major neurotransmitters that play an important role in analgesia by activating peripheral opioid receptors. Following the establishment of stable transfection of hMeSCs, the expressions of hPPE and GFP were confirmed and the production of methionine enkephalin (Met-enkephalin) was significantly increased compared to control naive hMeSCs $(p<0.05)$. Our in vitro data demonstrated that genetically engineered hMeSCs with transfected hPPE gene can constitutively produce opioid peptide Met-enkephalin at an augmented high level. hMeSCs are relatively easy to isolate from a patient's bone marrow aspirates and expand in culture by repeated passages. Autologous hMeSCs would not require immunosuppression when transplanted back into the same patient. Through targeted gene manipulation such as hPPE gene transfection, this may offer a virtually unlimited safe cell supply for the treatment of opioid-sensitive pain in humans.
\end{abstract}

Key words: Preproenkephalin; Gene transfection; Human mesenchymal stem cells; Autologous source; Enkephalins; Antinociception

\section{INTRODUCTION}

Previous studies have shown that transplantation of xenogeneic adrenal medullary tissue or isolated chromaffin cell suspensions into the spinal subarachnoid space produces antinociception in rats and in nonhuman primates $(7,17,18,20)$. Initial clinical trials using allogeneic adrenal chromaffin tissue provided pain relief in patients with terminal cancer $(4,11,26)$. Adrenal medulary chromaffin cells synthesize and release several compounds, including enkephalins and catecholamines $(5,23)$. These agents reduce pain sensitivity when injected directly into the spinal cord subarachnoid space $(7,16,17)$. However, availability of human adrenal tissue to serve as allografts for transplantation is very limited. Because effective therapy of a single patient requires large amounts of isolated chromaffin cells for transplantation, the clini- cal need for suitable sources of chromaffin cells is obvious. Bovine or porcine adrenal medullary tissue may provide alternative cell sources; however, these cells elicit host immunological response to the transplants $(2,7)$. Immunosuppression is necessary for the prolonged survival of the transplants, and immunosuppression may result in serious problems, including an increased susceptibility to tumor growth and infections. Although chromaffin cells are not very immunogenic and purified xenogeneic chromaffin cells can minimize immunorejection, even a small number of passenger cells (i.e., fibroblast and endothelial cells) in the transplant preparation are highly immunogenic $(2,10)$. In our experience with purified chromaffin cell transplants, a clear decrease in transplant function over time occurs (7). For example, with xenogeneic chromaffin cell transplants, the attenuation of pain may last only 3 weeks without 
immunosuppression (7), while analgesia can last longer (at least 5 weeks) with immunosuppression (20). The reasons for the gradual decline in analgesic effect are not yet clear; it may involve several factors in which immunorejection cannot be excluded, because the administration of immunosuppression itself enhances longer term graft survival and extends the analgesic effect $(7,9)$. In addition, there has been a concern regarding pathogen contamination of bovine spongiform encephalopathy for bovine chromaffin cell transplantation. Therefore, the ideal cell source would be autologous cells derived from the patient's own tissue.

With the advance of in vitro gene technology, there is a potential for genetically engineering the cells derived from a patient's own peripheral tissue for targeted clinical use $(12,13,21,24,25)$. Autologous human mesenchymal stem cells (hMeSCs) are relatively easy to isolate from bone marrow aspirates and then expand in culture by repeatable passages. These cells are favorable candidates for further genetic manipulation because they are able to proliferate and potentially differentiate into the cell lineage of various types including neural phenotypes and survive for prolonged period when transplanted into the CNS $(1,14,19,27)$. Preproenkephalin (PPE) is the precursor protein for enkephalins and enkephalin opioid peptides such as Met- and Leu-enkephalin, which are important neurotransmitters for inhibition of nociception $(3,5,6,15,25)$. Following transfection, the expression of human PPE (hPPE) and green fluorescent protein (GFP) genes and the production of Met-enkephalin in MeSCs were examined by RT-PCR and an immunoblot assay, respectively. Our results demonstrated that these genetically engineered hMeSCs have an enhanced hPPE gene expression and increased release of Metenkephalin in culture conditions. As a first step, we have successfully manipulated hMeSCs genetically to produce more opioid peptide Met-enkephalin by targeted gene transfection. hMeSCs are dividing cells and proliferate in culture with repeated passages. These autologous Met-enkephalin-producing hMeSCs may offer a virtually unlimited safe cell supply and have promise of serving as a useful alternative to adrenal chromaffin cells for the clinical treatment of opioid-sensitive pain.

\section{MATERIALS AND METHODS}

\section{Cell Cultures}

hMeSCs were purchased from Cambrex (Walkersville, MD), which were negative for CD11b, CD33, CD34, and CD133 antigens. hMeSCs were cultured in $75-\mathrm{cm}^{2}$ culture flasks (Corning, Cambridge, MA) in 20 $\mathrm{ml}$ of serum-supplemented growth medium consisting of Dulbecco's modified Eagle medium (Invitrogen, Carlsbad, CA), antibiotic-antimycotic mixture (Invitrogen), and FBS (Stem Cell Technologies, Vancouver, BC). The cells were incubated at $37^{\circ} \mathrm{C}$ in a $5 \% \quad \mathrm{CO}_{2}$ humidified incubation chamber (Fisher, Pittsburgh, PA). The cells were fed by replacing half the culture medium twice per week.

\section{Transfection of Human Preproenkephalin (hPPE)}

The hPPE cDNA (pGEMhPPE) was a gift from Dr. S. P. Wilson (University of South Carolina, School of Medicine, Department of Pharmacology, Physiology and Neuroscience). The hPPE cDNA was dissected out from pGEMhPPE by digestion with EcoRI and HindIII. The hPPE cDNA was purified on a gel (960 bp) and then ligated into EcoRI and HindIII site of pcDNA3.1(-)/Zeo (Invitrogen), human cytomegalovirus (CMV) promoterbased constitutive expression vectors [pcDNA3.1(-)/zeo/ hPPE]. To produce enhanced green fluorescent protein (EGFP) fusion hPPE, pcDNA3.1(-)/zeo/hPPE was digested by XhoI and HindIII and the resulting fragment (993 bp) was cloned into the pEGFP (BD Bioscience, Palo Alto, CA) XhoI and HindIII site (pEGFP/hPPE). All the clones were confirmed by DNA sequencing. Endotoxin-free plasmids are prepared by NucleoBond Plasmid EF Kit (BD Bioscience) from bacterial cultures. Transfection of the gene to the hMeSCs was performed by using NeuroPORTOR (Gene Therapy Systems, Inc., San Diego, CA) by following the protocol provided by the manufacturer. After the transfection, GFP expression was checked with a Leica fluorescent microscope.

\section{Gene Expression Analysis by RT-PCR}

Four days after transfection, hPPE and GFP gene expressions were analyzed by RT-PCR. RNAs were isolated from the cells using TRIzol reagent (Invitrogen) and treated with RNAase-free DNAase (Promega, Madison, WI). RT-PCR was performed using SuperScript One-Step RT-PCR with Platinum Taq (Invitrogen) using gene-specific primer sets: hPPE (5'-ACATCAACTTCC TGGCTTGCGT-3' and 5'-GCTCACTTCTTCCTCATT ATCA-3'), GFP (5'-CAAGGACGACGGCAACTACA AGACC-3' and 5'-GCGGACTGGGTGCTCAGGTAGT GGT-3'). DNA samples resulting from RT-PCR were analyzed on $2 \%$ E-Gel (Invitrogen).

\section{Immunoblot Assay for Met-Enkephalin in the Culture Media}

The serum-supplemented growth medium for hMeSCs was replaced by serum-free medium (DMEM with antibiotic-antimycotic) 1 day before Met-enkephalin assay. Twenty-four hours later, medium was collected and purified with YM-30 microcon (Millipore Corp., Bedford, MA). Filtered sample $(200 \mu \mathrm{l})$ and Met-enkephalin standards (50-1000 ng/slot, Phoenix Pharmaceuticals, Inc., Belmont, CA) were applied on Hybond ECL nitrocellurose membrane (Amersham Life Science, Piscataway, 
NJ) using the Slot Blot Hybridization Manifold (GENE Mate, Kaysville, UT). The membranes were blocked with 3\% normal donkey serum (Jackson ImmunoResearch, West Grove, PA) in phosphate-buffered saline containing $0.5 \%$ Tween 20 (PBST) for $2 \mathrm{~h}$ at room temperature. The membranes were then incubated with Met-enkephalin antibody made in rabbit (1:600, ImmunoStar Inc., Hudson, WI) for overnight at $4^{\circ} \mathrm{C}$. After washing with PBST three times, the membranes were incubated with anti-rabbit IgG peroxidase-linked species-specific whole donkey antibody (1:3000, Amersham Biosciences) for $90 \mathrm{~min}$ at room temperature. The membranes were washed with PBS, incubated with ECL Plus detection reagent (Amersham Biosciences) for $5 \mathrm{~min}$, and then exposed to Hyperfilm ECL (Amersham Biosciences). The films were developed, digitally scanned into a computer, and analyzed with NIH image (ImageJ, NIH). The data were normalized against cell numbers, which were counted using Bright-Line Hemacytometer (Hausser Scientific, Horsham, PA).

\section{RESULTS}

The hMeSCs transfected with GFP/hPPE fused gene were grown at $37^{\circ} \mathrm{C}$ for $24 \mathrm{~h}$ before in vitro analysis. These cells retained their ability to proliferate at a speed similar to that of the hMeSCs without gene transfection. Under the culture condition as mentioned above, these cells can double themselves by 7 days.

\section{RT-PCR Analysis}

Four days after transfection with GFP/hPPE fused gene, hMeSCs expressed both GFP and hPPE, which was analyzed by RT-PCR. As expected, the molecular size of the RT-PCR product for hPPE and GFP gene fragments was 425 and 318 bp, respectively. Naive hMeSCs expressed hPPE; however, transfection of GFP/ $\mathrm{hPPE}$ into hMeSCs greatly enhanced hPPE gene expression in these cells (Fig. 1).

\section{Production and Release of Met-Enkephalin}

The serum-supplemented growth medium for hMeSCs was replaced by serum-free medium (DMEM with antibiotic-antimycotic) 1 day before immunoblot assay for Met-enkephalin. Twenty-four hours later, medium was collected and purified for immunoblot assay. Naive $\mathrm{hMeSCs}$ were able to produce and release of Metenkephalin opioid peptide into the culture medium at a basal level of $1.731 \pm 0.124 \mathrm{pg} / \mathrm{cell} / 24 \mathrm{~h}$. Then we assessed whether the transgenic GFP/hPPE were able to drive the production and secretion of Met-enkephalin opioid peptide in the transfected hMeSC population. We found that the gene transfected hMeSCs had a significantly augmented production and release of Met-enkephalin opioid peptide at a rate of $2.42 \pm 0.46 \mathrm{pg} / \mathrm{cell} / 24 \mathrm{~h}$,

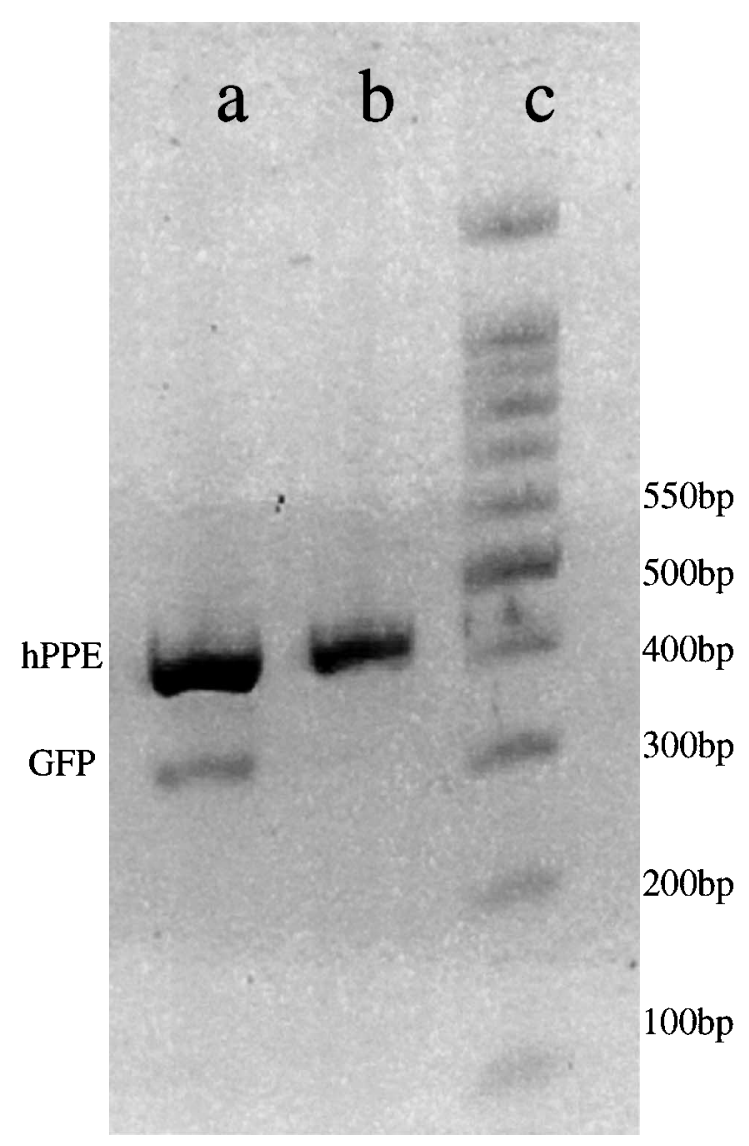

Figure 1. RT-PCR detection of GFP and hPPE expression in hMeSCs after gene transfection. Lane a: GFP and hPPE gene transfected hMeSCs showed GFP (318 bp) and hPPE expression (425 bp). Lane b: Nontransfected hMeSCs showed hPPE expression (425 bp) alone. Lane c: DNA ladder (100 bp).

when compared with naive hMeSCs $(p<0.05)$ (Fig. 2Fig. 2). Following transfection of GFP/hPPE/, GFP expression of hMeSCs was detected as green fluorescencelabeled cells under fluorescence microscope (Fig. 3).

\section{DISCUSSION}

Our present study demonstrates that hMeSCs transfected with a vector containing a fused hPPE and GFP gene were able to express both hPPE and GFP genes and constitutively produce a high level of Met-enkephalin opioid peptide in cell culture. As assessed by RTPCR and an immunoblot assay, our results reveal that naive hMeSCs have an inherent but weak PPE gene expression and are spontaneously able to release a basal level of Met-enkephalin at a rate of $1.731 \pm 0.124 \mathrm{pg} /$ cell/24 $\mathrm{h}$ in the cultured condition. Four days after hPPE gene transfection, hMeSCs obtained an enhanced hPPE expression and the production of Met-enkephalin significantly increased at a rate of $2.42 \pm 0.46 \mathrm{pg} / \mathrm{cell} / 24 \mathrm{~h}$ $(p<0.05)$ (Fig. 3). Therefore, genetic enhancement of 


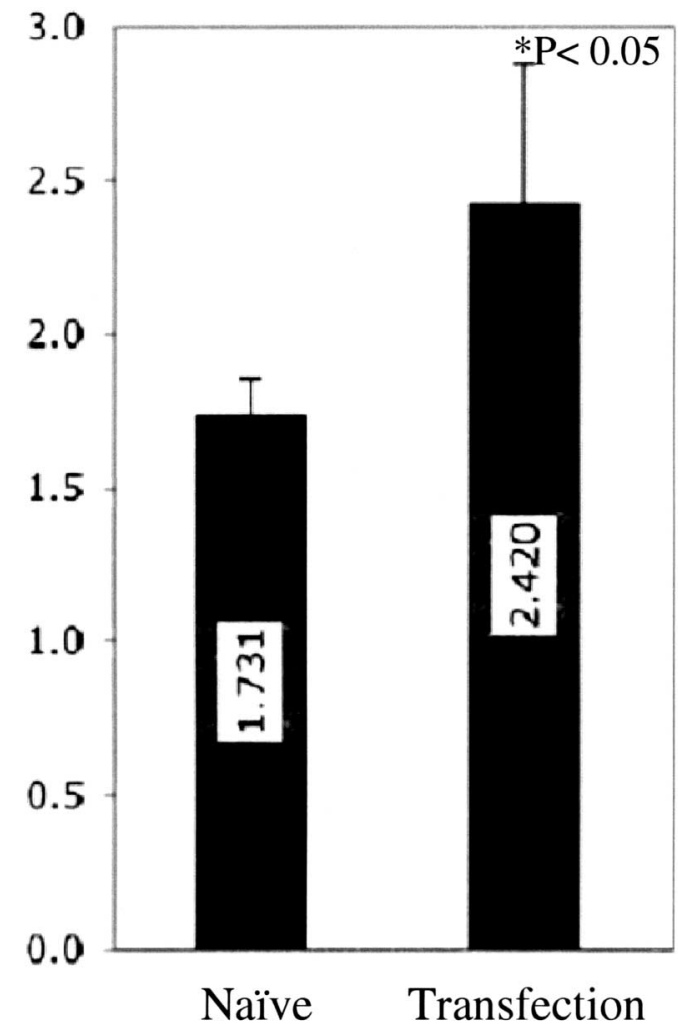

Figure 2. Met-enkephaline production of naive hMeSCs and hMeSCs transfected with EGFP/hPPE gene. Note the significantly increased met-enkephalin production in the culture of hMeSCs transfected with EGFP/hPPE gene $(* p<0.05)$.

hMeSCs by targeted hPPE gene transfection significantly increased their ability to produce more Metenkephalin. In accordance with naive MeSCs, the genetically enhanced Met-enkephalin-producing hMeSCs were stable and retained their ability to proliferate in vitro, suggesting that these cells are both viable and functional. Thus, we have successfully generated expandable hMeSCs into Met-enkephalin opioid peptideproducing cells, which can release Met-enkephlin at the similar levels to normally found in the primary chromaffin cells.

We have previously briefly described a similar approach of hPPE gene transfection into human neuroncommitted teratocarcinoma (NT-2) cells (8). These gene transfected NT-2 cells expressed hPPE gene and produce Met-enkephalin. A subpopulation of them can be terminally differentiated into neurons by retinoic acid treatment. When transplanted intrathecally into a rat pain model, the differentiated neuronal cells derived from NT-2 cells transfected with hPPE gene demonstrated antinociception at an efficacy similar to that observed with chromaffin cell transplantation (8). The reduced pain sensitivity is obviously mediated by opioid peptides released by the transplanted cells because it can be blocked by intrathecal administration of the opioid antagonist naloxone. These results suggest that the genetically engineered cells expressing hPPE and producing Met-enkephalin have promise to serve as a useful alternative to adrenal chromaffin cells in pain treatment.

As reported previously, transplantation of xenogenic adrenal medullary tissue or isolated medullary chromaffin cell suspensions into the pain modulatory regions in the CNS can produce robust antinociceptive effect in animal pain models $(16,18,20)$. Preliminary clinical trials using allogenic adrenal medullary tissue produced a long-term analgesic effect in patients with refractory cancer pain without development of tolerance $(4,11,26)$. Beside the limited availability of allogeneic adrenal tissue and the lack of tissue for individual transplantation, there is a concern regarding immunorejection and pathogen contamination for adrenal tissues of xenogeneic sources $(2,7)$. It would be ideal to develop a new source of autologous cells derived from the patient's own peripheral tissue because the cells of an autologous origin would not require immune suppression when transplanted back to the same patient. Our present study demonstrated that hMeSCs could be a favorable autologous cell candidate for generating chromaffin cell-like cells through further gene manipulation because these cells inherently express hPPE gene and are able to spontaneously release low level of opioid peptide Met-enkephalin.

Wilson and colleagues and others $(3,6,15,25)$ have introduced the hPPE gene to sensory neurons by a herpes-mediated gene delivery system that successfully modulates certain types of nociceptive reactions in experimental pain models. In an effort to develop a new source of chromaffin-like cells from an autologous source of cells, we genetically transfected hMeSCs with a mammalian expression vector containing a fused gene of hPPE and enhanced GFP and successfully generated hMeSCs opioid peptide Met-enkephalin-producing cells without viral delivery. Because autologous hMeSCs are easily isolated from patients' own peripheral tissues and are expandable in cell culture for further gene manipulation, our approach may provide a favorable alternative to adrenal chromaffin cell transplantation in the treatment of opioid-sensitive chronic pain, a treatment that will not cause host immune rejection and thereby improving analgesic efficacy. By avoiding the use of viral vectors as a vehicle to deliver a target gene into pain modulatory regions, the strategies of in vitro gene manipulation of autologous cells may hold the promise of providing safer and more efficacious approaches for targeted clinical application.

The hMeSCs are able to proliferate and have the potential to differentiate into the cell lineage of various 


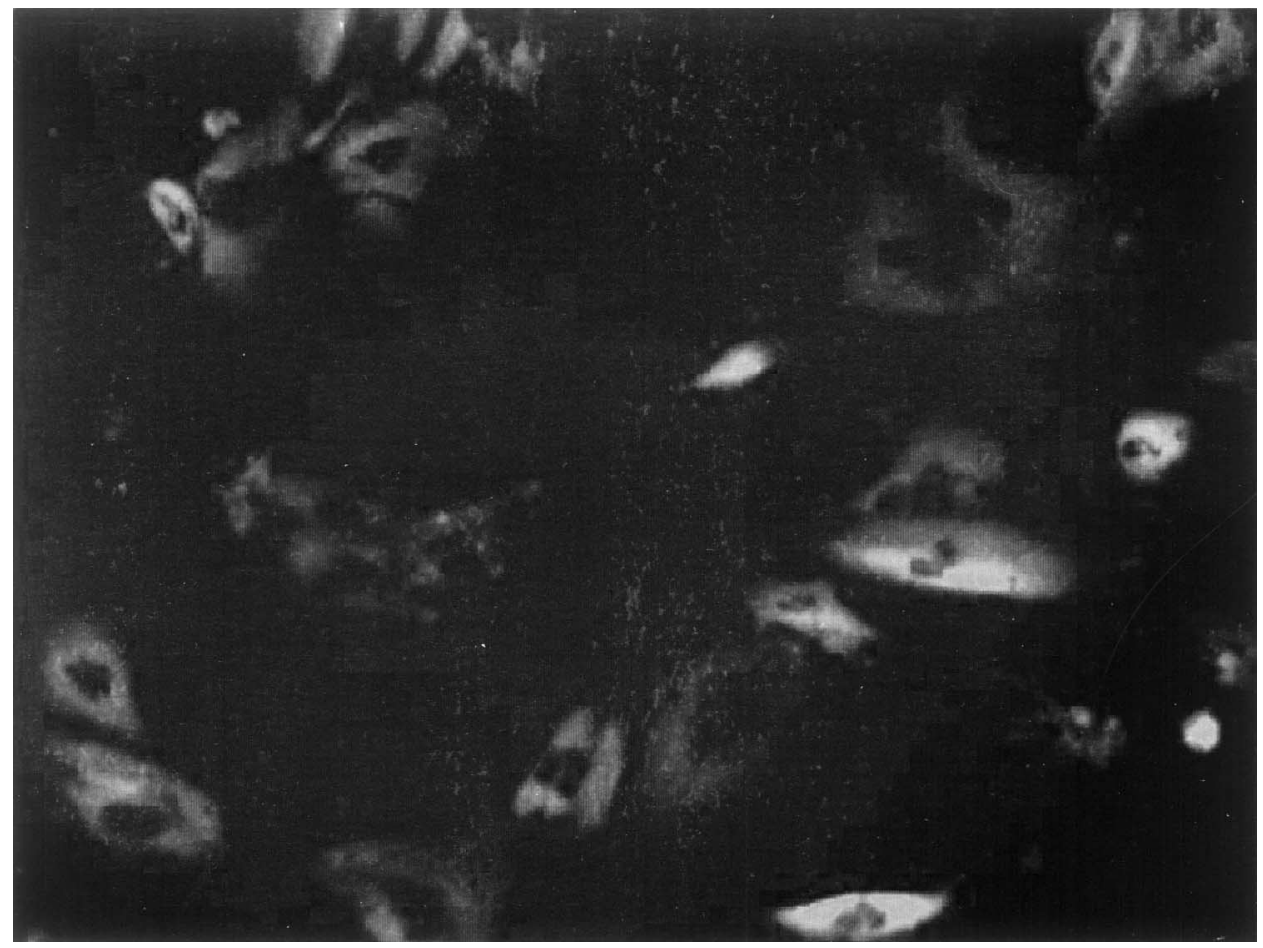

Figure 3. Picture showing the enhanced green fluorescent protein (EGFP) expression in the cytoplasm of the hMeSCs transfected with a fused gene of EGFP/hPPE under the fluorescence microscope $(\times 400)$.

types, including the ability of acquiring neural phenotypes both in vitro and in vivo and surviving for prolonged periods when transplanted into the brain $(1,14$, 19,27), suggesting the feasibility of hMeSCs for CNS transplantation. After gene transfection, these cells retained the capacity for proliferation and differentiation. These properties of hMeSCs make them valuable when they are used as vehicles for the release of neuroactive substances into the host CNS environment and function as a biological pump, providing an endogenous source of renewable neuroactive substances.

Unlike chromaffin cells, which are postmitotic, hMeSCs are self-renewal stem cells. Because these genetically engineered $\mathrm{hMeSCs}$ can multiply in culture over repeated passages, this source of cells may offer a sufficient cell supply for the need of transplantation. Our in vitro data suggest that the bone marrow-derived hPEE gene transfected MeSCs may provide a valuable cell source to serve as an alternative to adrenal chromaffin cells in pain treatment. However, further work should be performed in different animal pain models to evaluate the analgesic effect of these Met-enkaphalin-producing $\mathrm{hMeSCs}$ in vivo before clinical use.

In summary, the present study is an initial step in the development of chromaffin-like cells from autologous hMeSCs. Our results provide the first in vitro experi- mental data about the therapeutic potential of autologous hMeSCs transfected by hPPE gene in pain treatment. We demonstrated that naive hMeSCs have a low level of endogenous hPPE gene expression and low level of Met-enkephalin production and that gene-enhanced hMeSCs by hPPE transfection are able to constitutively produce Met-enkephalin and spontaneously release Metenkephalin at a high level in the cell culture. The hPPE expression was confirmed by RT-PCR, and the level of Met-enkephalin production was examined by an immunoblot assay. The data obtained from our studies suggest that hMeSCs transfected with hPPE gene without viral delivery may serve as a suitable alternative to adrenal chromaffin cells for transplantation to control chronic pain.

ACKNOWLEDGMENTS: This study was supported by NIH grants R01 DA 015511 to G.D.P and R01 AG 23472 to K.S.

\section{REFERENCES}

1. Cogle, C. R.; Yachnis, A. T.; Laywell, E. D.; Zander, D. S.; Wingard, J. R.; Steindler, D. A.; Scott, E. W. Bone marrow transdifferentiation in brain after transplantation: A retrospective study. Lancet 363:1432-1437; 2004.

2. Czech, K. A.; Ryan, J. W.; Sagen, J.; Pappas, G. D. The influence of xenotransplant immunogenicity and immunosuppression on host MHC expression in the rat CNS. Exp. Neurol. 147(1):66-83; 1997. 
3. Kang, W.; Wilson, M. A.; Bender, M. A.; Glorioso, J. C.; Wilson, S. P. Herpes virus-mediated preproenkephalin gene transfer to the amygdala is antinociceptive. Brain Res. 792:133-135; 1998 .

4. Lazorthes, Y.; Sagen, J.; Sallerin, B.; Tkaczuk, J.; Duplan, H.; Sol, J. C.; Tafani, M.; Bes, J. C. Human chromaffin cell graft into the CSF for cancer pain managemant: A prospective phase II clinical study. Pain 87:19-32; 2000.

5. Livett, B. G.; Dean, D. M.; Whelan, L. G.; Udenfriend, S.; Rossier, J. Co-release of enkephalin and catecholamines from cultured adrenal chromaffin cells. Nature 289(5795):317-319; 1981.

6. Liu, F.; Housley, P. R.; Wilson, S. P. Initial processing of human proenkephalin in bovine chromaffin cells. J. Neurochem. 67:1457-1462; 1996.

7. Lu, Y.; Jing, R.; Yeomans, D. C.; Pappas, G. D. Porcine chromaffin cells, culture, and transplant for antinociceptive effects in rodents and primates. Neurol. Res. 26(7): 707-712; 2004.

8. Lu, Y.; Sugaya, I.; Zhang, Z. J.; Qu, T. Y.; Pappas, G. D.; Wilson, S. P.; Sugaya, K. Antinociceptive effect of intrathecal transplantation of preproenkephalin encoding neural progenitor cells in rat. Chicago Chapter of SFN (abstract); 2005.

9. Ortega, J. D.; Sagen, J.; Pappas, G. D. Short-term immunosuppression enhances long-term survival of bovine chromaffin cell xenografts in rat CNS. Cell Transplant. 1(1):33-41; 1992.

10. Pappas, G. D. Fine structure of host-graft relationships between transplanted chromaffin cells and CNS. FASEB J. 13(Suppl. 2):S277-280; 1999.

11. Pappas, G. D.; Lazorthes, Y.; Bes, J. C.; Tafani, M.; Winnie, A. P. Relief of intractable cancer pain by human chromaffin cell transplants: Experience at two medical centers. Neurol. Res. 19:71-77; 1997.

12. Pereboeva, L.; Komarova, S.; Mikheeva, G.; Krasnykh, V.; Curiel, D. T. Approaches to utilize mesenchymal progenitor cells as cellular vehicles. Stem Cells 21:389-404; 2003.

13. Polakiewicz, R. D.; Behar, O. Z.; Comb, M. J.; Rosen, H. Regulation of proenkephalin expression in cultured skin mesenchymal cells. Mol. Endocrinol. 6:399-408; 1992.

14. Qu, T. Y.; Dong, X. J.; Sugaya, I.; Vaghani, A.; Pulido, J.; Sugaya, K. Bromodeoxyuridine increases multipotency of human bone marrow-derived stem cells. Restor. Neurol. Neurosci. 22(6):459-468; 2004.

15. Rosen, H.; Krichevsky, A.; Polakiewicz, R. D.; Benzakine, S.; Bar-Shavit, Z. Developmental regulation of proen- kephalin gene expression in osteoblasts. Mol. Endocrinol. 9:1621-1631; 1995.

16. Sagen, J.; Pappas, G. D.; Pollard, H. B. Analgesia induced by isolated bovine chromaffin cells implanted in rat spinal cord. Proc. Natl. Acad. Sci. USA 83(19):7522-7526; 1986.

17. Sagen, J.; Kemmler, J. E.; Wang, H. Adrenal medullary transplants increase spinal cord cerebrospinal fluid catecholamine levels and reduce pain sensitivity. J. Neurochem. 56(2):623-627; 1991.

18. Sagen, J.; Wang, H.; Pappas, G. D. Adrenal medullary implants in the rat spinal cord reduce nociception in a chronic pain model. Pain 42(1):69-79; 1990.

19. Sanchez-Ramos, J.; Song, S.; Cardozo-Pelaez, F.; Hazzi, C.; Stedeford, T.; Willing, A.; Freeman, T. B.; Saporta, S.; Janssen, W.; Patel, N.; Cooper, D. R.; Sanberg, P. R. Adult bone marrow stromal cells differentiate into neural cells in vitro. Exp. Neurol. 164(2):247-256; 2000.

20. Sol, J. C.; Sallerin, B.; Larrue, S.; Li, R. Y.; Jozan, S.; Tortosa, F.; Mascott, C.; Carraoue, F.; Tafani, M.; Lazorthes, Y. Intrathecal xenogeneic chromaffin cell grafts reduce nociceptive behavior in a rodent tonic pain model. Exp. Neurol. 186(2):198-211; 2004.

21. Sugiyama, O.; Orimo, H.; Suzuki, S.; Yamashita, K.; Ito, H.; Shimada, T. Bone formation following transplantation of genetically modified primary bone marrow stromal cells. J. Orthop. Res. 21(4):630-637; 2003.

22. Unsicker, K. The chromaffin cell: Paradigm in cell, developmental and growth factor biology. J. Anat. 183:207$221 ; 1993$.

23. Wilson, S. P.; Chang, K. J.; Viveros, O. H. Proportional secretion of opioid peptides and catecholamines from adrenal chromaffin cells in culture. J. Neurosci. 2(8):1150$1156 ; 1982$.

24. Wilson, S. P.; Yeomans, D. C. Genetic therapy for pain management. Curr. Rev. Pain 4(6):445-450; 2000.

25. Wilson, S. P.; Yeomans, D. C. Virally mediated delivery of enkephalin and other neuropeptide transgenes in experimental pain models. Ann. NY Acad. Sci. 971:515-521; 2002.

26. Winnie, A. P.; Pappas, G. D.; Das Gupta, T. K.; Wang, H.; Ortega, J. D.; Sagen, J. Subarachnoid adrenal medullary transplants for terminal cancer pain. A report of preliminary studies. Anesthesiology 79(4):644-653; 1993.

27. Woodbury, D.; Schwarz, E. J.; Prockop, D. J.; Black, I. B. Adult rat and human bone marrow stromal cells differentiate into neurons. J. Neurosci. Res. 61(4):364-370; 2000 . 